PROCEEDINGS OF THE

AMERICAN MATHEMATICAL SOCIETY

Volume 131, Number 10, Pages 3241-3249

S 0002-9939(03)06869-2

Article electronically published on February 12, 2003

\title{
COVERING FOR CATEGORY AND TRIGONOMETRIC THIN SETS
}

\author{
PETER ELIAŠ
}

(Communicated by Carl G. Jockusch, Jr.)

\begin{abstract}
In this work we consider several combinatorial principles satisfied for cardinals smaller than $\operatorname{cov}(\mathcal{M})$, the covering number of the ideal of first category sets on real line. Using these principles we prove that there exist $\mathrm{N}_{0^{-}}$ sets (similarly N-sets, A-sets) which cannot be covered by fewer than $\operatorname{cov}(\mathcal{M})$ pD-sets (A-sets, N-sets, respectively). This improves the results of our previous paper (1997).
\end{abstract}

The study of special sets of reals related to the convergence of trigonometric series, generally called 'trigonometric thin sets', was one of the classical topics of harmonic analysis (see e.g. 4]). Recently, many investigations were made concerning the set-theoretic properties of such sets. A serious interest turns on the cardinal characteristics of structures related to trigonometric thin sets (see e.g. [3, [5], 6], [8], [10, [11]).

This paper has two parts. In the first part we present two new combinatorial characterizations of the cardinal $\operatorname{cov}(\mathcal{M})$, the covering number of the ideal of first category sets (Corollary 5]. We use these characterizations in the second part to prove some theorems about trigonometric thin sets (Theorems 12, 13, and 16).

\section{Combinatorics}

We use the standard set-theoretic notation. Hence, $\omega$ is the set of all natural numbers, $[\omega]^{<\omega}$ and $[\omega]^{\omega}$ denote the sets of all finite and infinite subsets of $\omega$, $A^{B}$ is the set of all functions from $B$ to $A$, and $|A|$ is the cardinality of a set $A$. Quantifiers $\exists^{\infty}$ and $\forall^{\infty}$ stand for 'there are infinitely many' and 'for all but finitely many', respectively.

Let $\mathcal{M}$ denote the ideal of first category (meager) sets on real line. Let us recall that $\operatorname{cov}(\mathcal{M})$, the covering number of $\mathcal{M}$, is the minimum size of a family $\mathcal{F} \subseteq \mathcal{M}$ such that $\bigcup \mathcal{F}=\mathbb{R}$. We will use the following combinatorial characterizations of this cardinal, due to Bartoszyński ([1], [2]).

Lemma 1. Let $\kappa$ be a cardinal. The following conditions are equivalent:

(1) $\kappa<\operatorname{cov}(\mathcal{M})$,

Received by the editors October 23, 2001 and, in revised form, May 17, 2002.

2000 Mathematics Subject Classification. Primary 03E75; Secondary 03E17, 40A30, 43A46.

Key words and phrases. Covering, first category sets, meager sets, trigonometric thin sets, A-sets, N-sets, $\mathrm{N}_{0}$-sets, pD-sets.

The work on this paper was supported by grant 1/7555/20 of Slovak Grant Agency VEGA.

(C)2003 American Mathematical Society 
(2) for every family $\mathcal{F} \subseteq \omega^{\omega},|\mathcal{F}| \leq \kappa$, there exists a function $g \in \omega^{\omega}$ such that $(\forall f \in \mathcal{F})\left(\exists^{\infty} n\right) g(n)=f(n)$

(3) for every family $\mathcal{F} \subseteq \omega^{\omega},|\mathcal{F}| \leq \kappa$, and for every family $\mathcal{X} \subseteq[\omega]^{\omega},|\mathcal{X}| \leq \kappa$, there exists $g \in \omega^{\omega}$ such that $(\forall f \in \mathcal{F})(\forall X \in \mathcal{X})\left(\exists^{\infty} n \in X\right) g(n)=f(n)$,

(4) for every family $\mathcal{F} \subseteq \omega^{\omega},|\mathcal{F}| \leq \kappa$, there exists a function $S \in([\omega]<\omega)^{\omega}$, such that $(\forall n)|S(n)|=n+1$, and $(\forall f \in \mathcal{F})\left(\exists^{\infty} n\right) f(n) \in S(n)$.

We will add two more conditions to this list. First, we need to prove some lemmas.

Lemma 2. For every family $\mathcal{F} \subseteq[\omega]^{\omega},|\mathcal{F}|<\operatorname{cov}(\mathcal{M})$, and for every $h \in \omega^{\omega}$, there exists $g \in \omega^{\omega}$ such that $(\forall n) g(n+1) \geq h(g(n))$, and $(\forall X \in \mathcal{F})|\operatorname{rng}(g) \cap X|=\omega$.

Proof. Let $\mathcal{F}=\left\{X_{\alpha}: \alpha<\kappa\right\}, \kappa<\operatorname{cov}(\mathcal{M})$. Without loss of generality, we assume that $h$ is non-decreasing and $h(n)>n$ for all $n$.

Put $k_{0}=0, k_{n+1}=h\left(k_{n}\right)$ for $n \in \omega$. For $\alpha<\kappa$ and $n \in \omega$, let $f_{\alpha}(n) \in \omega$ be such that

$$
\left|\left\{j: j<f_{\alpha}(n) \wedge\left[k_{j}, k_{j+1}\right) \cap X_{\alpha} \neq \emptyset\right\}\right|=3 n+1 .
$$

Put $f_{\alpha}^{*}(n)=\left[0, k_{f_{\alpha}(n)}\right) \cap X_{\alpha}$. Since $f_{\alpha}^{*}$-s can be coded as elements of $\omega^{\omega}$, by Lemma 1 there exists $g^{*}$ such that $(\forall \alpha<\kappa)\left(\exists^{\infty} n\right) g^{*}(n)=f_{\alpha}^{*}(n)$. Moreover, we may assume that $(\forall n)(\exists \alpha<\kappa) g^{*}(n)=f_{\alpha}^{*}(n)$. Thus for each $n$,

$$
\left|\left\{j:\left[k_{j}, k_{j+1}\right) \cap g^{*}(n) \neq \emptyset\right\}\right|=3 n+1,
$$

and we can take $m_{n} \in g^{*}(n)$ such that for every $i<n$ and $j \in \omega$, if $m_{i} \in\left[k_{j}, k_{j+1}\right)$, then $m_{n} \notin\left[k_{j-1}, k_{j+2}\right)$.

Let $g \in \omega^{\omega}$ be an increasing function such that $\operatorname{rng}(g)=\left\{m_{n}: n \in \omega\right\}$. For every $n$, if $g(n) \in\left[k_{j}, k_{j+1}\right)$, then $\left[k_{j+1}, k_{j+2}\right) \cap \operatorname{rng}(g)=\emptyset$, and thus $g(n+1) \geq$ $k_{j+2}=h\left(k_{j+1}\right) \geq h(g(n))$, since $k_{j+1}>g(n)$. Moreover, if $g^{*}(n)=f_{\alpha}^{*}(n)$, then $m_{n} \in X_{\alpha}$. Hence $\operatorname{rng}(g) \cap X_{\alpha}$ is infinite for every $\alpha<\kappa$.

Lemma 3. Let $\mathcal{F} \subseteq \omega^{\omega},|\mathcal{F}|<\operatorname{cov}(\mathcal{M})$, and let every $f \in \mathcal{F}$ be increasing. Then for every $h \in \omega^{\omega}$ there exists $g \in \omega^{\omega}$ such that $(\forall n) g(n+1) \geq h(g(n))$, and $(\forall f \in \mathcal{F})\left(\exists^{\infty} n\right)(\exists k \leq n) g(k)=f(n)$.

Proof. By Lemma 1, there exists $g^{\prime}$ such that $(\forall f \in \mathcal{F})\left(\exists^{\infty} n\right) g^{\prime}(n)=f(n)$. Moreover, we may assume that $(\forall n)(\exists f \in \mathcal{F}) g^{\prime}(n)=f(n)$, and hence $g^{\prime}(n) \geq n$ for all $n$. For $f \in \mathcal{F}$, put $X_{f}=\left\{n: g^{\prime}(n)=f(n)\right\}$ and $h^{*}(n)=\max \left\{n+1, h\left(g^{\prime}(n)\right)\right\}$. By Lemma 2 there exists $g^{*} \in \omega^{\omega}$ such that $(\forall n) g^{*}(n+1) \geq h^{*}\left(g^{*}(n)\right)$, and $(\forall f \in \mathcal{F})\left|\operatorname{rng}\left(g^{*}\right) \cap X_{f}\right|=\omega$. Clearly $g^{*}$ is increasing.

For $n \in \omega$, let $g(n)=g^{\prime}\left(g^{*}(n)\right)$. Then for every $n, g(n+1)=g^{\prime}\left(g^{*}(n+1)\right) \geq$ $g^{*}(n+1) \geq h^{*}\left(g^{*}(n)\right) \geq h\left(g^{\prime}\left(g^{*}(n)\right)\right)=h(g(n))$. Moreover, if $n \in \operatorname{rng}\left(g^{*}\right) \cap X_{f}$, then $n=g^{*}(k)$ for some $k \leq n$, and $g(k)=g^{\prime}\left(g^{*}(k)\right)=g^{\prime}(n)=f(n)$.

Lemma 4. Let $\mathcal{F} \subseteq \omega^{\omega},|\mathcal{F}|<\operatorname{cov}(\mathcal{M})$. Then there exists a function $g \in \omega^{\omega}$ such that $(\forall n)(\exists f \in \mathcal{F}) g(n+1)=f(g(n))$, and $(\forall f \in \mathcal{F})\left(\exists^{\infty} n\right) g(n+1)=f(g(n))$.

Proof. First, we show the existence of $g^{\prime} \in \omega^{\omega}$ such that $(\forall n)(\exists f \in \mathcal{F}) g^{\prime}(n+1) \geq$ $f\left(g^{\prime}(n)\right)$, and $(\forall f \in \mathcal{F})\left(\exists^{\infty} n\right) g^{\prime}(n+1) \geq f\left(g^{\prime}(n)\right)$. Using Lemma 1, we can find $h \in \omega^{\omega}$ such that $h(n)>n$ for all $n$, and the set $X_{f}=\{n: f(n) \leq h(n)\}$ is infinite for every $f \in \mathcal{F}$. By Lemma 2, there exists $g^{\prime} \in \omega^{\omega}$ such that for all $n, g^{\prime}(n+1) \geq h\left(g^{\prime}(n)\right)$, and for all $f \in \mathcal{F},\left|\operatorname{rng}\left(g^{\prime}\right) \cap X_{f}\right|=\omega$. Moreover, we may assume that $(\forall n)(\exists f \in \mathcal{F}) g^{\prime}(n) \in X_{f}$. Since $g^{\prime}$ is increasing, we have $(\forall f \in$ 
$\mathcal{F})\left(\exists^{\infty} n\right) g^{\prime}(n) \in X_{f}$. Clearly, if $g^{\prime}(n) \in X_{f}$, then $f\left(g^{\prime}(n)\right) \leq h\left(g^{\prime}(n)\right) \leq g^{\prime}(n+1)$. Thus $g^{\prime}$ is as we have expected.

For $f \in \mathcal{F}, n \in \omega$, denote $f^{*}(n)=\max \{f(0), \ldots, f(n)\}$. As we have just proved, there exists $g^{*} \in \omega^{\omega}$ such that for all $f \in \mathcal{F}$, the set $Y_{f}=\left\{n: g^{*}(n+1) \geq f^{*}\left(g^{*}(n)\right)\right\}$ is infinite, and $\bigcup_{f \in \mathcal{F}} Y_{f}=\omega$.

For given $f \in \mathcal{F}$ and $n \in \omega$, put $H_{f}(n)=f \nmid\left\{0, \ldots, g^{*}(n)\right\}$. Since $H_{f}(n)$-s can be coded as elements of $\omega$, by Lemma 1 there exists a function $H$ such that $(\forall f \in \mathcal{F})\left(\exists^{\infty} n \in Y_{f}\right) H(n)=H_{f}(n)$. Without loss of generality we may assume also that $(\forall n)(\exists f \in \mathcal{F})\left(n \in Y_{f} \wedge H(n)=H_{f}(n)\right)$.

Put $g(0)=0, g(n+1)=H(n)(g(n))$. For every $n$, we have $H(n)=H_{f}(n)$ for some $f \in \mathcal{F}$ such that $n \in Y_{f}$, and hence if $k \in \operatorname{dom}(H(n))$, then $k \leq g^{*}(n)$, and $H(n)(k)=H_{f}(n)(k)=f(k) \leq f^{*}\left(g^{*}(n)\right) \leq g^{*}(n+1)$. Thus $H(n)(k) \in$ $\operatorname{dom}(H(n+1))$, and the definition of $g$ makes sense. Moreover, if $H(n)=H_{f}(n)$, then $g(n+1)=H_{f}(n)(g(n))=f(g(n))$. Hence $g$ has the required properties.

Corollary 5. Let $\kappa$ be a cardinal. The following conditions are equivalent to (1)-(4) from Lemma 1:

(5) for every family $\mathcal{F} \subseteq \omega^{\omega},|\mathcal{F}| \leq \kappa$, such that every $f \in \mathcal{F}$ is increasing, and for every $h \in \omega^{\omega}$, there exists $g \in \omega^{\omega}$ such that $(\forall n) g(n+1) \geq h(g(n))$, and $(\forall f \in \mathcal{F})\left(\exists^{\infty} n\right)(\exists k \leq n) g(k)=f(n)$,

(6) for every family $\mathcal{F} \subseteq \omega^{\omega},|\mathcal{F}| \leq \kappa$, there exists $g \in \omega^{\omega}$ such that $(\forall n)(\exists f \in$ $\mathcal{F}) g(n+1)=f(g(n))$, and $(\forall f \in \mathcal{F})\left(\exists^{\infty} n\right) g(n+1)=f(g(n))$.

Proof. As we have shown in the previous two lemmas, (1) implies both (5) and (6). We will prove that (5) implies (4), and (6) implies (2).

Assume that (5) holds. Let $\mathcal{F} \subseteq \omega^{\omega},|\mathcal{F}| \leq \kappa$, and let $h(n)=n+1$ for all $n \in \omega$. Clearly if $g \in \omega^{\omega}$ satisfies the conditions of (5), then $g$ is increasing, and the function $S$ such that $S(n)=\{g(0), \ldots, g(n)\}, n \in \omega$, satisfies the condition of (4). Thus (5) implies (4).

Let $\mathcal{F} \subseteq \omega^{\omega},|\mathcal{F}| \leq \kappa$, and let (6) hold true. Fix a bijection $p \in(\omega \times \omega)^{\omega}$. For $f \in \mathcal{F}$, let $f^{*} \in \omega^{\omega}$ be such that for all $k, l \in \omega, f^{*}(p(k, l))=p(k+1, f(k))$. By (6), there exists $g^{*} \in \omega^{\omega}$ such that $(\forall n)(\exists f \in \mathcal{F}) g^{*}(n+1)=f^{*}\left(g^{*}(n)\right)$, and $(\forall f \in \mathcal{F})\left(\exists^{\infty} n\right) g^{*}(n+1)=f^{*}\left(g^{*}(n)\right)$. For $n \in \omega$, let $k_{n}, l_{n}$ be such that $g^{*}(n)=p\left(k_{n}, l_{n}\right)$. Now, if $g^{*}(n+1)=f^{*}\left(g^{*}(n)\right)$ for some $f \in \mathcal{F}$ and $n \in \omega$, then $p\left(k_{n+1}, l_{n+1}\right)=f^{*}\left(p\left(k_{n}, l_{n}\right)\right)=p\left(k_{n}+1, f\left(k_{n}\right)\right)$. It follows that for all $n$, $k_{n}=n+k_{0}$ and $f\left(k_{n}\right)=l_{n+1}$. If we put $g(n)=0$ for $n<k_{0}$, and $g\left(n+k_{0}\right)=l_{n+1}$ for all $n \in \omega$, then $g$ will satisfy the condition of (2). Thus (6) implies (2).

An open problem is whether the condition from Lemma2 2 is equivalent to (1)-(6). It can be formulated as follows.

Problem 6. Let $\kappa$ be the minimum cardinality of a family $\mathcal{F} \subseteq[\omega]^{\omega}$ for which there exists $h \in \omega^{\omega}$ such that for all $g \in \omega^{\omega}$, if $(\forall n) g(n+1) \geq h(g(n))$, then $(\exists X \in \mathcal{F})|\operatorname{rng}(g) \cap X|<\omega$. Is it consistent that $\kappa>\operatorname{cov}(\mathcal{M})$ ?

\section{TRIGONOMETRIC THIN SETS}

From many types of trigonometric thin sets, we will consider the following four: $\mathrm{N}$-sets, $\mathrm{N}_{0}$-sets, A-sets, and pD-sets. A set $X$ of real numbers is called an $N$-set if there exists a trigonometric series absolutely converging on $X$ while not absolutely converging everywhere, or equivalently, if there exists a sequence of positive reals 
$\left\{a_{n}\right\}_{n=1}^{\infty}$ such that $\sum_{n=1}^{\infty} a_{n}=\infty$, and for all $x \in X, \sum_{n=1}^{\infty} a_{n}|\sin \pi n x|<\infty$. It is called an $N_{0}$-set (or an $A$-set, a $p D$-set) if there exists an increasing sequence of natural numbers $\left\{n_{k}\right\}_{k=0}^{\infty}$ such that for all $x \in X, \sum_{k=0}^{\infty}\left|\sin \pi n_{k} x\right|<\infty$ (or $\lim _{k \rightarrow \infty}\left|\sin \pi n_{k} x\right|=0,\left|\sin \pi n_{k} x\right| \leq 2^{-k}$ for almost all $k$, respectively). Let us note that in the definitions above, we can use the distance of $x$ to the nearest integer, i.e. the function $\|x\|=\min \{x-k: k \in \mathbb{Z}\}$, instead of the function $|\sin \pi x|$. More about these types of sets can be found, e.g., in [6], 7].

We denote by $\mathcal{N}, \mathcal{N}_{0}, \mathcal{A}$, and $p \mathcal{D}$ the families of all N-sets, $\mathrm{N}_{0}$-sets, A-sets, and pD-sets, respectively. Directly from the definitions we can see that $\mathcal{N}_{0} \subseteq \mathcal{N}$, $\mathcal{N}_{0} \subseteq \mathcal{A}$, and $p \mathcal{D} \subseteq \mathcal{N}_{0}$. In [9], S. Kahane showed that there exist $\mathrm{N}_{0}$-sets which cannot be covered by countably many pD-sets, N-sets which cannot be covered by countably many A-sets, and A-sets which cannot be covered by countably many $\mathrm{N}$-sets. In [7] we have proved that 'countably many' can be replaced by 'fewer than $\operatorname{add}(\mathcal{M})^{\prime}$, where $\operatorname{add}(\mathcal{M})$ is the additivity of the ideal $\mathcal{M}$ of all first category sets. Now, we will improve these results to 'fewer than $\operatorname{cov}(\mathcal{M})$ ' (Theorems 1213. and 16). Let us note that it is consistent with ZFC that $\operatorname{add}(\mathcal{M})<\operatorname{cov}(\mathcal{M})$; the consistency is established by the Cohen model (see e.g. [2]).

By an interval we will mean a set of the form $\{x \in \mathbb{R}: a \leq x \leq b\}$, for some $a<b$. Thus an interval will always be compact and have non-empty interior. The length of an interval $I$, denoted by $\lambda(I)$, is the value $b-a$.

Lemma 7. Let $n \geq 1,0<\varepsilon \leq 1$. For every interval $I$ such that $\lambda(I)=1 / n$ there exists an interval $J \subseteq I$ such that $\|n x\| \leq \varepsilon$ for all $x \in J$, and $\lambda(J)=\varepsilon / n$.

Proof. Since $\lambda(I)=1 / n$, there exists $x_{0} \in I$ such that $\left\|n x_{0}\right\|=0$. Let $J$ be any sub-interval of $I$ such that $x_{0} \in J$ and $\lambda(J)=\varepsilon / n$.

Lemma 8. Let $n \geq 1,0<\varepsilon<1 / 2$. For every interval I such that $\lambda(I)>2 \varepsilon / n$ there exists an interval $J \subseteq I$ such that $\|n x\| \geq \varepsilon$ for all $x \in J$, and

$$
\lambda(J)=\min \left\{\frac{\lambda(I)}{2}-\frac{\varepsilon}{n}, \frac{1-2 \varepsilon}{n}\right\} .
$$

Proof. Assume that $I=[a-b, a+b]$, thus $b=\lambda(I) / 2>\varepsilon / n$. If $\left\|n x_{0}\right\|=0$ for some $x_{0} \in[a-b-\varepsilon / n, a]$, then let us take

$$
J=\left[x_{0}+\frac{\varepsilon}{n}, x_{0}+\min \left\{b, \frac{1-\varepsilon}{n}\right\}\right] .
$$

Clearly $J \subseteq I, \lambda(J)=\min \{b-\varepsilon / n,(1-2 \varepsilon) / n\}$, and if $x \in J$, then $\varepsilon / n \leq$ $\left|x-x_{0}\right| \leq(1-\varepsilon) / n$, thus $\|n x\| \geq \varepsilon$. Similarly, if $\left\|n x_{0}\right\|=0$ for some $x_{0} \in$ $[a, a+b+\varepsilon / n]$, then we can take

$$
J=\left[x_{0}-\min \left\{b, \frac{1-\varepsilon}{n}\right\}, x_{0}-\frac{\varepsilon}{n}\right] .
$$

Finally, if $\|n x\|>0$ for all $x \in[a-b-\varepsilon / n, a+b+\varepsilon / n]$, then clearly $\|n x\| \geq \varepsilon$ for all $x \in I$. We can take arbitrary interval $J \subseteq I$ with $\lambda(J)=\min \{b-\varepsilon / n,(1-2 \varepsilon) / n\}$.

Corollary 9. Let $1 \leq n \leq n^{\prime}$. For every interval I such that $\lambda(I)=1 / n$, there exists an interval $J \subseteq I$ such that $\lambda(J)=1 / n^{\prime}$, and if $x \in J$, then $\|n x\| \leq n / n^{\prime}$.

Proof. Use Lemma 7 with $\varepsilon=n / n^{\prime}$. 
Corollary 10. Let $1 \leq n \leq 2 m \leq n^{\prime}, 0<\varepsilon \leq 1 / 4$, and $n / n^{\prime} \leq \varepsilon$. For every interval $I$ such that $\lambda(I)=1 / n$, there exists an interval $J \subseteq I$ such that $\lambda(J)=$ $1 / n^{\prime}$, and if $x \in J$, then $\|n x\| \leq 4 \varepsilon$, and $\|m x\| \geq \varepsilon / 2$.

Proof. By Lemma 7 there exists an interval $I^{\prime} \subseteq I$ such that $\|n x\| \leq 4 \varepsilon$ for all $x \in I^{\prime}$, and $\lambda\left(I^{\prime}\right)=4 \varepsilon / n$. By Lemma 8 there exists an interval $J^{\prime} \subseteq I^{\prime}$ such that $\|m x\| \geq \varepsilon / 2$ for all $x \in J^{\prime}$, and

$$
\lambda\left(J^{\prime}\right)=\min \left\{\frac{2 \varepsilon}{n}-\frac{\varepsilon}{2 m}, \frac{1-\varepsilon}{m}\right\} \geq \min \left\{\frac{\varepsilon}{n}, \frac{1}{2 m}\right\} \geq \frac{1}{n^{\prime}} .
$$

Let $J$ be any interval such that $J \subseteq J^{\prime}$ and $\lambda(J)=1 / n^{\prime}$.

Lemma 11. Let $\left\{n_{k}\right\}_{k \in \omega},\left\{m_{i}\right\}_{i \in \omega}$ be increasing sequences of natural numbers, $\left\{\varepsilon_{i}\right\}_{i \in \omega}$ be a sequence of reals, and $k \in \omega^{\omega}$ be such that for all $i$,

(1) $n_{k(i)} \leq 2 m_{i}<n_{k(i)+1}$,

(2) $k(i+1)>k(i)$

(3) $n_{k(i)} / n_{k(i)+1} \leq \varepsilon_{i} \leq 1 / 4$.

Then there exists a real $x$ such that

(4) for all $k,\left\|n_{k} x\right\| \leq \begin{cases}4 \varepsilon_{i} & \text { if } k=k(i) \text { for some } i, \\ n_{k} / n_{k+1} & \text { if } k \notin\{k(i): i \in \omega\},\end{cases}$

(5) for all $i,\left\|m_{i} x\right\| \geq \varepsilon_{i} / 2$.

Proof. We take $x \in \bigcap_{k \in \omega} I_{k}$ where $\left\{I_{k}\right\}_{k \in \omega}$ is a nested sequence of intervals defined as follows. Let $I_{0}$ be such that $\lambda\left(I_{0}\right)=1 / n_{0}$. For every $k$, assume that $\lambda\left(I_{k}\right)=1 / n_{k}$. If $k \notin\{k(i): i \in \omega\}$, then by Corollary 9 there exists an interval $I_{k+1} \subseteq I_{k}$ such that $\lambda\left(I_{k+1}\right)=1 / n_{k+1}$ and $\left\|n_{k} x\right\| \leq n_{k} / n_{k+1}$ for all $x \in I_{k+1}$. If $k=k(i)$ for some $i$, then by Corollary 10 there exists $I_{k+1} \subseteq I_{k}$ such that $\lambda\left(I_{k+1}\right)=1 / n_{k+1}$, and if $x \in I_{k+1}$, then $\left\|n_{k} x\right\| \leq 4 \varepsilon_{i}$, and $\left\|m_{i} x\right\| \geq \varepsilon_{i} / 2$.

Theorem 12. Let $\left\{n_{k}\right\}_{k \in \omega}$ be an increasing sequence of natural numbers such that $\sum_{k \in \omega} n_{k} / n_{k+1}<\infty$. Then the $N_{0}$-set $\left\{x: \sum_{k \in \omega}\left\|n_{k} x\right\|<\infty\right\}$ cannot be covered by a union of fewer than $\operatorname{cov}(\mathcal{M})$ pD-sets.

Proof. Let $\left\{X_{\alpha}\right\}_{\alpha<\kappa}$ be a sequence of $\mathrm{pD}$-sets, $\kappa<\operatorname{cov}(\mathcal{M})$. We will find a real $x$ such that $\sum_{k \in \omega}\left\|n_{k} x\right\|<\infty$ and $x \notin \bigcup_{\alpha<\kappa} X_{\alpha}$.

Fix a monotone sequence of positive reals $\left\{\varepsilon_{j}\right\}_{j \in \omega}$ such that $\sum \varepsilon_{j}<\infty$, and $\varepsilon_{j} \leq 1 / 4$ for all $j$. Find an increasing function $k^{*} \in \omega^{\omega}$ such that if $j \geq k^{*}(i)$, then $n_{j} / n_{j+1} \leq \varepsilon_{i}$, and put $h(i)=n_{k^{*}(i+1)}$. For $\alpha<\kappa$, let $\left\{m_{j}^{\alpha}\right\}_{j \in \omega}$ be an increasing sequence of natural numbers such that $\left(\forall x \in X_{\alpha}\right)\left(\forall^{\infty} j\right)\left\|m_{j}^{\alpha} x\right\|<\varepsilon_{j} / 2$ and $m_{0}^{\alpha} \geq$ $n_{k^{*}(0)} / 2$. By Lemma 3 there exists $g \in \omega^{\omega}$ such that $(\forall i) g(i+1) \geq h(g(i))$, and $(\forall \alpha<\kappa)\left(\exists^{\infty} j\right)(\exists i \leq j) g(i)=2 m_{j}^{\alpha}$. Without a loss of generality we may assume that $(\forall i)(\exists \alpha<\kappa)(\exists j \geq i) g(i)=2 m_{j}^{\alpha}$, thus every $g(i)$ is even. Put $m_{i}=g(i) / 2$.

Let $k \in \omega^{\omega}$ be such that for all $i, n_{k(i)} \leq 2 m_{i}<n_{k(i)+1}$. Clearly $m_{0} \geq n_{k^{*}(0)} / 2$, and thus $k(0) \geq k^{*}(0)$. We have $g(i+1) \geq n_{k^{\prime}}>g(i)$, where $k^{\prime}=k^{*}(g(i)+1)$, hence $k(i+1) \geq k^{\prime}>k(i)$. Since $g$ is increasing, we have $k^{\prime} \geq k^{*}(i+1)$. Thus for all $i \in \omega, k(i) \geq k^{*}(i)$, and hence $n_{k(i)} / n_{k(i)+1} \leq \varepsilon_{i}$.

Now, for a real $x$ obtained from Lemma[11] we have $\sum_{k \in \omega}\left\|n_{k} x\right\| \leq \sum_{i \in \omega} 4 \varepsilon_{i}+$ $\sum_{k \in \omega} n_{k} / n_{k+1}<\infty$, and for every $\alpha<\kappa$ there exist infinitely many $j$-s such that for some $i \leq j,\left\|m_{j}^{\alpha} x\right\|=\left\|m_{i} x\right\| \geq \varepsilon_{i} / 2 \geq \varepsilon_{j} / 2$, thus $x \notin \bigcup_{\alpha<\kappa} X_{\alpha}$. 
Theorem 13. Let $\left\{n_{k}\right\}_{k \in \omega}$ be an increasing sequence of natural numbers and let $\left\{\varrho_{k}\right\}_{k \in \omega}$ be a sequence of non-negative reals such that $\lim _{k \rightarrow \infty} \varrho_{k}=0, \sum_{k \in \omega} \varrho_{k}=$ $\infty$, and $\sum_{k \in \omega} \varrho_{k} n_{k} / n_{k+1}<\infty$. Then the $N$-set $\left\{x: \sum_{k \in \omega} \varrho_{k}\left\|n_{k} x\right\|<\infty\right\}$ cannot be covered by a union of fewer than $\operatorname{cov}(\mathcal{M})$ A-sets.

Proof. Put $K=\left\{k: n_{k} / n_{k+1} \leq 1 / 4\right\}$. Since $\sum_{k \in \omega} \varrho_{k} n_{k} / n_{k+1}<\infty$, we have $\sum_{k \notin K} \varrho_{k}<\infty$, and since $\sum_{k \in \omega} \varrho_{k}=\infty$, the set $K$ is infinite. For $j \in \omega$, let $k_{j}$ denote its $j$-th element. Clearly $\lim _{j \rightarrow \infty} \varrho_{k_{j}}=0, \sum_{j \in \omega} \varrho_{k_{j}}=\infty$, and for every $x, \sum_{k \in \omega} \varrho_{k}\left\|n_{k} x\right\|<\infty$ iff $\sum_{j \in \omega} \varrho_{k_{j}}\left\|n_{k_{j}} x\right\|<\infty$. Moreover, $n_{k_{j}} / n_{k_{j+1}} \leq$ $n_{k_{j}} / n_{k_{j}+1} \leq 1 / 4$ for all $j \in \omega$. Hence by taking $\left\{n_{k_{j}}\right\}_{j \in \omega}$ instead of $\left\{n_{k}\right\}_{k \in \omega}$ we can ensure that $n_{k} / n_{k+1} \leq 1 / 4$ for all $k \in \omega$.

Let $k^{*} \in \omega^{\omega}$ be an increasing function such that for all $i$, if $j \geq k^{*}(i)$, then $\varrho_{j} \leq 2^{-i}$. Let $\left\{X_{\alpha}\right\}_{\alpha<\kappa}$ be a sequence of A-sets, $\kappa<\operatorname{cov}(\mathcal{M})$. For every $\alpha<\kappa$, let $\left\{m_{j}^{\alpha}\right\}_{j \in \omega}$ be an increasing sequence of natural numbers such that $m_{0}^{\alpha} \geq n_{k^{*}(0)}$, and for all $x \in X_{\alpha}, \lim _{j \rightarrow \infty}\left\|m_{j}^{\alpha} x\right\|=0$.

Similarly as in Theorem 12 there exists an increasing sequence of natural numbers $\left\{m_{i}\right\}_{i \in \omega}$ and an increasing function $k \in \omega^{\omega}$ such that for all $i, n_{k(i)} \leq 2 m_{i}<$ $n_{k(i)+1}$ and $k(i) \geq k^{*}(i)$ and thus $\varrho_{k(i)} \leq 2^{-i}$. By putting $\varepsilon_{i}=1 / 4$ in Lemma 11] we can find a real $x$ such that $\left\|n_{k} x\right\| \leq n_{k} / n_{k+1}$ for all $k \notin\{k(i): i \in \omega\}$, and $\left\|m_{i} x\right\| \geq 1 / 8$ for all $i$. Clearly $\sum_{k \in \omega} \varrho_{k}\left\|n_{k} x\right\| \leq \sum_{i \in \omega} 2^{-i}+\sum_{k \in \omega} \varrho_{k} n_{k} / n_{k+1}<\infty$, and for every $\alpha<\kappa$ there exist infinitely many $j$-s such that for some $i,\left\|m_{j}^{\alpha} x\right\|=$ $\left\|m_{i} x\right\| \geq 1 / 8$, thus $x \notin \bigcup_{\alpha<\kappa} X_{\alpha}$.

The following lemma is a reformulation of Lemma 2.9 in 7 .

Lemma 14. Let $\left\{\varrho_{n}\right\}_{n \in \omega}$ be a sequence of non-negative reals, let $\alpha \leq \beta, 0<\theta \leq$ $1 / 7$, and let $I$ be an interval such that $\lambda(I) \geq 3 \theta / \alpha$. Then there exists an interval $J \subseteq I$ such that $\lambda(J) \geq \theta / \beta$, and if $x \in J$, then

$$
\sum_{\alpha \leq n \leq \beta} \varrho_{n}\|n x\| \geq \frac{\theta}{8} \sum_{\alpha \leq n \leq \beta} \varrho_{n} .
$$

Proof. At first let us consider the case $\beta \leq 3 \alpha$. There exists $x_{0}$ such that

$$
\left[x_{0}-\frac{\theta}{2 \beta}, x_{0}+\frac{3 \theta}{2 \alpha}+\frac{\theta}{2 \beta}\right] \subseteq I .
$$

Let $K=\left\{n \in \omega: \alpha \leq n \leq \beta \wedge\left\|n x_{0}\right\| \geq \theta\right\}, L=\left\{n \in \omega: \alpha \leq n \leq \beta \wedge\left\|n x_{0}\right\|<\theta\right\}$.

If $\sum_{n \in K} \varrho_{n} \geq \frac{1}{2} \sum_{n \in K \cup L} \varrho_{n}$, put $J=\left[x_{0}-\frac{\theta}{2 \beta}, x_{0}+\frac{\theta}{2 \beta}\right]$. Then for $x \in J$ and $n \in K$ we obtain $\|n x\| \geq\left\|n x_{0}\right\|-n\left|x-x_{0}\right| \geq \theta-\theta / 2=\theta / 2$, and thus

$$
\sum_{n \in K \cup L} \varrho_{n}\|n x\| \geq \frac{\theta}{4} \sum_{n \in K \cup L} \varrho_{n}
$$

Otherwise, we have $\sum_{n \in L} \varrho_{n} \geq \frac{1}{2} \sum_{n \in K \cup L} \varrho_{n}$. Put $J=\left[x_{0}+\frac{3 \theta}{2 \alpha}, x_{0}+\frac{3 \theta}{2 \alpha}+\frac{\theta}{\beta}\right]$. For $x \in J$ and $n \in L$ we obtain $3 \theta / 2 \leq n\left|x-x_{0}\right| \leq 11 \theta / 2 \leq 1-3 \theta / 2$, and thus $\|n x\| \geq\left\|n\left(x-x_{0}\right)\right\|-\left\|n x_{0}\right\| \geq 3 \theta / 2-\theta=\theta / 2$. Hence (1) holds true again.

In the case that $\beta>3 \alpha$, define a sequence $\alpha_{0}<\alpha_{1}<\cdots<\alpha_{k}$ as follows: put $\alpha_{0}=\alpha, \alpha_{i+1}=3 \alpha_{i}$ for $0 \leq i<k$, and $\alpha_{k}=\beta \leq 3 \alpha_{k-1}$. Let $K^{\prime}=\left\{n \in \omega: \alpha_{i} \leq\right.$ $n \leq \alpha_{i+1}$ for some $i$ even $\}, L^{\prime}=\left\{n \in \omega: \alpha_{i} \leq n \leq \alpha_{i+1}\right.$ for some $i$ odd $\}$. 
If $\sum_{n \in K^{\prime}} \varrho_{n} \geq \frac{1}{2} \sum_{n \in K^{\prime} \cup L^{\prime}} \varrho_{n}$, find a sequence of intervals $I \supseteq I_{0} \supseteq I_{2} \supseteq \ldots$ such that for every $i, 0 \leq i \leq k, i$ even,

$$
\begin{gathered}
\lambda\left(I_{i}\right) \geq \frac{\theta}{\alpha_{i+1}} \geq \frac{3 \theta}{\alpha_{i+2}}, \text { and } \\
\sum_{\alpha_{i} \leq n \leq \alpha_{i+1}} \varrho_{n}\|n x\| \geq \frac{\theta}{4} \sum_{\alpha_{i} \leq n \leq \alpha_{i+1}} \varrho_{n} \text { for all } x \in I_{i} .
\end{gathered}
$$

Similarly, if $\sum_{n \in L^{\prime}} \varrho_{n} \geq \frac{1}{2} \sum_{n \in K^{\prime} \cup L^{\prime}} \varrho_{n}$, find a sequence of intervals $I \supseteq I_{1} \supseteq I_{3} \supseteq$ $\ldots$ such that (2) and (3) hold true for every $i, 0 \leq i \leq k, i$ odd. In both cases, let $J$ be the last interval in the sequence, i.e. $I_{k}$ if $k$ is even, $I_{k-1}$ if $k$ is odd.

Lemma 15. Let $\left\{n_{k}\right\}_{k \in \omega}$ be an increasing sequence of natural numbers and let $\left\{\varrho_{n}\right\}_{n \in \omega}$ be a sequence of non-negative reals such that

$$
\lim _{k \rightarrow \infty} \frac{n_{k}}{n_{k+1}}=0, \text { and } \sum_{n \in \omega} \varrho_{n}=\infty .
$$

Let $k^{\prime}, \varepsilon \leq 1$ be such that $n_{k} / n_{k+1} \leq \varepsilon$ for all $k \geq k^{\prime}$. Then for every interval I such that $\lambda(I)=1 / n_{k^{\prime}}$ there exist $k^{\prime \prime}>k^{\prime}$ and an interval $J \subseteq I$ such that $\lambda(J)=1 / n_{k^{\prime \prime}}$, and for all $x \in J,\left\|n_{k} x\right\| \leq \varepsilon$ whenever $k^{\prime} \leq k<k^{\prime \prime}$, and

$$
\sum_{n_{k^{\prime}} \leq n<n_{k^{\prime \prime}}} \varrho_{n}\|n x\| \geq 1
$$

Proof. For given reals $\alpha, \beta$, we denote

$$
S(\alpha, \beta)=\sum_{\alpha \leq n \leq \beta} \varrho_{n} .
$$

We put $S(\alpha, \beta)=0$ if there is no $n \in \omega$ such that $\alpha \leq n \leq \beta$.

Let $\gamma=1 / 7$. We will consider two cases.

(a) There exists $\delta \geq \gamma$ such that $\sum_{k \in \omega} S\left(\gamma n_{k}, \delta n_{k}\right)=\infty$. Then we can find a sequence $\left\{\theta_{k}\right\}_{k \in \omega}$ such that $\lim _{k \rightarrow \infty} \theta_{k}=0, \sum_{k \in \omega} \theta_{k} S\left(\gamma n_{k}, \delta n_{k}\right)=\infty$, and for all $k, \theta_{k} \geq \delta n_{k} / n_{k+1}$. Put $\alpha_{k}=\gamma n_{k}, \beta_{k}=\delta n_{k}$, for $k \in \omega$.

(b) For all $\delta \geq \gamma$, we have $\sum_{k \in \omega} S\left(\gamma n_{k}, \delta n_{k}\right)<\infty$. Then for all $\delta \geq \gamma$, we must have $\sum_{k \in \omega} S\left(\delta n_{k}, \gamma n_{k+1}\right)=\infty$. Thus there exists a sequence $\left\{\delta_{k}\right\}_{k \in \omega}$ such that $\lim _{k \rightarrow \infty} \delta_{k}=\infty, \sum_{k \in \omega} S\left(\delta_{k} n_{k}, \gamma n_{k+1}\right)=\infty$, and for all $k, \gamma<\delta_{k} \leq \gamma n_{k+1} / n_{k}$. Put $\alpha_{k}=\delta_{k} n_{k}, \beta_{k}=\gamma n_{k+1}, \theta_{k}=\gamma$, for $k \in \omega$.

As can be easily checked, in both cases there exists $k_{0}$ such that for all $k \geq k_{0}$,

$$
\alpha_{k} \leq \beta_{k}<\alpha_{k+1}, 0<\theta_{k} \leq 1 / 7, \varepsilon / n_{k} \geq 3 \theta_{k} / \alpha_{k}, \text { and } \theta_{k} / \beta_{k} \geq 1 / n_{k+1} .
$$

Moreover, we have $\sum_{k \in \omega} \theta_{k} S\left(\alpha_{k}, \beta_{k}\right)=\infty$.

Find $i \geq \max \left\{k_{0}, k^{\prime}\right\}$ such that $\alpha_{i} \geq n_{k^{\prime}}$. There exists $j \geq i$ such that

$$
\sum_{i \leq k \leq j} \theta_{k} S\left(\alpha_{k}, \beta_{k}\right) \geq 8
$$

Put $k^{\prime \prime}=j+1$. Clearly $\beta_{j} \leq \theta_{j} n_{k^{\prime \prime}}<n_{k^{\prime \prime}}$.

Let us define a nested sequence of intervals $\left\{I_{k}\right\}_{k^{\prime} \leq k<k^{\prime \prime}}$ as follows. Put $I_{k^{\prime}}=I$. We will always have $\lambda\left(I_{k}\right)=1 / n_{k}$. By Lemma 7, there exists an interval $J_{k} \subseteq I_{k}$ such that $\lambda\left(J_{k}\right)=\varepsilon / n_{k}$, and if $x \in J_{k}$, then $\left\|n_{k} x\right\| \leq \varepsilon$. If $k<i$, let $I_{k+1}$ be any interval such that $I_{k+1} \subseteq J_{k}$ and $\lambda\left(I_{k+1}\right)=1 / n_{k+1}$. For $i \leq k \leq j$, we have 
$\lambda\left(J_{k}\right) \geq 3 \theta_{k} / \alpha_{k}$, and hence by Lemma 14 there exists an interval $J_{k}^{\prime} \subseteq J_{k}$ such that $\lambda\left(J_{k}^{\prime}\right) \geq \theta_{k} / \beta_{k}$, and if $x \in J_{k}^{\prime}$, then

$$
\sum_{\alpha_{k} \leq n \leq \beta_{k}} \varrho_{n}\|n x\| \geq \theta_{k} S\left(\alpha_{k}, \beta_{k}\right) / 8 .
$$

Let $I_{k+1}$ be any interval such that $I_{k+1} \subseteq J_{k}^{\prime}$ and $\lambda\left(I_{k+1}\right)=1 / n_{k+1}$.

Put $J=I_{k^{\prime \prime}}$. For $x \in J$, we have $\left\|n_{k} x\right\| \leq \varepsilon$ whenever $k^{\prime} \leq k<k^{\prime \prime}$, and

$$
\sum_{n_{k^{\prime}} \leq n<n_{k^{\prime \prime}}} \varrho_{n}\|n x\| \geq \sum_{i \leq k \leq j} \sum_{\alpha_{k} \leq n \leq \beta_{k}} \varrho_{n}\|n x\| \geq \sum_{i \leq k \leq j} \theta_{k} S\left(\alpha_{k}, \beta_{k}\right) / 8 \geq 1 .
$$

Using this lemma we can prove the last theorem.

Theorem 16. Let $\left\{n_{k}\right\}_{k \in \omega}$ be an increasing sequence of natural numbers such that $\lim _{k \rightarrow \infty} n_{k} / n_{k+1}=0$. Then the A-set $\left\{x: \lim _{k \rightarrow \infty}\left\|n_{k} x\right\|=0\right\}$ cannot be covered by a union of fewer than $\operatorname{cov}(\mathcal{M}) N$-sets.

Proof. Let $\left\{I_{i}\right\}_{i \in \omega}$ be an enumeration of all intervals of the form $\left[m / n_{k},(m+1) / n_{k}\right]$, with integer $m$. Let $\left\{X_{\alpha}\right\}_{\alpha<\kappa}$ be a sequence of N-sets, $\kappa<\operatorname{cov}(\mathcal{M})$. We will find a real $x$ such that $\lim _{k \rightarrow \infty}\left\|n_{k} x\right\|=0$ and $x \notin \bigcup_{\alpha<\kappa} X_{\alpha}$.

For every $\alpha<\kappa$, let $\left\{\varrho_{n}^{\alpha}\right\}_{n \in \omega}$ be a sequence of non-negative reals such that $\sum_{n \in \omega} \varrho_{n}^{\alpha}=\infty$, and for all $x \in X_{\alpha}, \sum_{n \in \omega} \varrho_{n}^{\alpha}\|n x\|<\infty$. We will define a function $f_{\alpha} \in \omega^{\omega}$ as follows.

For given $i \in \omega$, let $k^{\prime}$ be such that $\lambda\left(I_{i}\right)=1 / n_{k^{\prime}}$. Put $\varepsilon=\max \left\{n_{k} / n_{k+1}\right.$ : $\left.k \geq k^{\prime}\right\}$. By Lemma 15, there exist $k^{\prime \prime}>k^{\prime}$ and an interval $J \subseteq I_{i}$ such that $\lambda(J)=1 / n_{k^{\prime \prime}}$, and for all $x \in J,\left\|n_{k} x\right\| \leq \varepsilon$ whenever $k^{\prime} \leq k<k^{\prime \prime}$, and

$$
\sum_{n_{k^{\prime}} \leq n<n_{k^{\prime \prime}}} \varrho_{n}^{\alpha}\|n x\| \geq 1 .
$$

Let $k^{*} \geq k^{\prime \prime}$ be such that $n_{k^{*}} / n_{k^{*}+1} \leq \varepsilon / 2$. There exists an interval $J^{*} \subseteq J$ such that for all $x \in J^{*},\left\|n_{k} x\right\| \leq n_{k} / n_{k+1}$ whenever $k^{\prime \prime} \leq k<k^{*},\left\|n_{k^{*}} x\right\| \leq \varepsilon$, and $\lambda\left(J^{*}\right)=\varepsilon / n_{k^{*}} \geq 2 / n_{k^{*}+1}$. Hence we can find $I_{j} \subseteq J^{*}$ such that $\lambda\left(I_{j}\right)=1 / n_{k^{*}+1}$. We put $f(i)=j$.

By Lemma 目, there exists $g \in \omega^{\omega}$ such that $(\forall j)(\exists \alpha<\kappa) g(j+1)=f_{\alpha}(g(j))$ and $(\forall \alpha<\kappa)\left(\exists^{\infty} j\right) g(j+1)=f_{\alpha}(g(j))$. For $j \in \omega$, let $k(j)$ be such that $\lambda\left(I_{g(j)}\right)=$ $1 / n_{k(j)}$. Clearly $I_{g(j+1)} \subseteq I_{g(j)}$ and $k(j+1)>k(j)$ for all $j$, and if we put $\varepsilon_{j}=\max \left\{n_{k} / n_{k+1}: k \geq k(j)\right\}$, then $\lim _{j \rightarrow \infty} \varepsilon_{j}=0$. Let us take $x \in \bigcap_{j \in \omega} I_{g(j)}$. We have $\left\|n_{k} x\right\| \leq \varepsilon_{j}$ whenever $k \geq k(j)$, and thus $\lim _{k \rightarrow \infty}\left\|n_{k} x\right\|=0$. Moreover, if $g(j+1)=f_{\alpha}(g(j))$, then

$$
\sum_{n_{k(j)} \leq n<n_{k(j+1)}} \varrho_{n}^{\alpha}\|n x\| \geq 1
$$

and hence for all $\alpha<\kappa, \sum_{n \in \omega} \varrho_{n}^{\alpha}\|n x\|=\infty$, and thus $x \notin X_{\alpha}$.

We finish with an open problem.

Problem 17. Is it possible to replace the cardinal $\operatorname{cov}(\mathcal{M})$ in Theorems 12,13 and 16 by some consistently greater cardinal? 


\section{REFERENCES}

[1] Bartoszyński, T., Combinatorial aspects of measure and category, Fund. Math. 127 (1987), 225-239. MR 88m:04001

[2] Bartoszyński, T., Judah, H., Set theory. On the structure of the real line, A K Peters, Ltd., Wellesley, MA, 1995. MR 96k:03002

[3] Bartoszyński, T., Scheepers, M., Remarks on sets related to trigonometric series, Topology Appl. 64 (1995), 133-140. MR 96h:42004

[4] Bary, N. K., A treatise on trigonometric series, The Macmillan Co., New York, 1964. MR 30:1347

[5] Bukovská, Z., Bukovský, L., Adding small sets to an N-set, Proc. Amer. Math. Soc. 123 (1995), 3867-3873. MR 96b:04002

[6] Bukovský, L., Kholshchevnikova, N. N., Repický, M., Thin sets of harmonic analysis and infinite combinatorics, Real Anal. Exchange 20 (1994/95), 454-509. MR 97b:43004

[7] Eliaš, P., A classification of trigonometrical thin sets and their interrelations, Proc. Amer. Math. Soc. 125 (1997), 1111-1121. MR 97f:43006

[8] Kada, M., Slalom, prediction, and permitted trigonometric thin sets, preprint.

[9] Kahane, S., Antistable classes of thin sets in harmonic analysis, Illinois J. Math. 37 (1993), 186-223. MR 94g:43005

[10] Laflamme, C., Combinatorial aspects of $\mathrm{F}_{\sigma}$ filters with an application to $\mathcal{N}$-sets, Proc. Amer. Math. Soc. 125 (1997), 3019-3025. MR 97m:04003

[11] Repický, M., Towers and permitted trigonometric thin sets, Real Anal. Exchange 21 (1995/96), 648-655. MR 97f:42013

Mathematical Institute, Slovak Academy of Sciences, Grešákova 6, 04001 Košice, SLOVAKIA

E-mail address: elias@kosice.upjs.sk 